\title{
A spectroscopic survey of FHB stars near the south galactic pole $\dagger$
}

\author{
Silvia Rossi ${ }^{1}$, Roberto Ortiz ${ }^{2}$, Ronald Wilhelm ${ }^{3}$, Roberto Costa ${ }^{1}$ \\ \& Timothy C. Beers ${ }^{4}$ \\ ${ }^{1}$ Departamento de Astronomia, IAG/USP, Rua do Matão 1226 Cidade Universitaria, \\ 05508-900, São Paulo, SP, Brazil \\ ${ }^{2}$ Escola de Artes, Ciencias e Humanidades, USP, Av. Arlindo Bettio, 1000 \\ 03828-000, São Paulo, SP, Brazil \\ ${ }^{3}$ Department of Physics, Campus Box 41051, Texas Tech University Lubbock
} TX 79409, USA

\begin{abstract}
${ }^{4}$ Department of Physics and Astronomy, CSCE: Center for the Study of Cosmic Evolution, and JINA: Joint Institute for Nuclear Astrophysics, Michigan State University, East Lansing, MI 48824-1116, USA
\end{abstract}

\begin{abstract}
We present the results of a medium-resolution spectroscopic survey of 43 field horizontal-branch (FHB) candidates carried out near the south galactic pole, selected from the original list of FHB candidates compiled by Beers et al. (2007). The observation list includes only stars classified as "high-probability" candidates, according to their 2MASS infrared colours. Atmospheric parameters of some stars have been obtained by comparing some spectral features with theoretical models provided by Kurucz (1993). A comparison between the grid of model atmospheres with some parameters of the $\mathrm{H} \delta$ line allowed the determination of $\log \mathrm{g}$, whilst $[\mathrm{Fe} / \mathrm{H}]$ was estimated by the equivalent widths of the MgII 4481 and CaII lines. About $77 \%$ of the sample have been classified as FHB stars, $10 \%$ as subdwarfs, whilst the remaining $13 \%$ are probably main-sequence A-type stars far from the Galactic plane.
\end{abstract}

Keywords. stars: horizontal-branch, Galaxy: halo, Galaxy: stellar content

\section{The Definition of the Sample and Observations}

This survey is an extension of the study carried out by Ortiz et al. (2007), who studied a sample of 44 stars near the south galactic pole with $\mathrm{T}_{\mathrm{J}-\mathrm{H}}$ and $\mathrm{T}_{\mathrm{J}-\mathrm{K}}>7900 \mathrm{~K}$. In the present study, the sample consists of 43 stars, selected according to the criteria proposed by Beers et al. (2007), especially defined to minimize the contamination by main-sequence stars. The criterium that defines a "high-probability" FHB is: $-0.2<(\mathrm{B}-$ $\mathrm{V})_{o}<+0.2$. The spectra were obtained in September 2007 with the Boller \& Chivens Cassegrain Spectrograph attached to the 1.6-m Telescope of the Pico dos Dias Observatory (LNA, southeast Brazil). The reciprocal dispersion was $1.2 \AA /$ pixel, using a 600 lines/mm grating, and the SNR varied between $19 / 1$ and $125 / 1$ at $\lambda=4480 \AA$.

\section{Results}

Some characteristics of the Balmer lines can help in the classification of these stars. For example, the $\mathrm{D}_{0.2}$ and $\mathrm{R}$ parameters, examined jointly, distinguish subdwarfs among FHB and main-sequence Av stars (Pier 1983, Sommer-Larsen \& Christensen 1986, Beers

$\dagger$ Based on observations obtained at the Pico dos Dias Observatory, operated by the National Laboratory for Astrophysics (LNA), Brazil. 

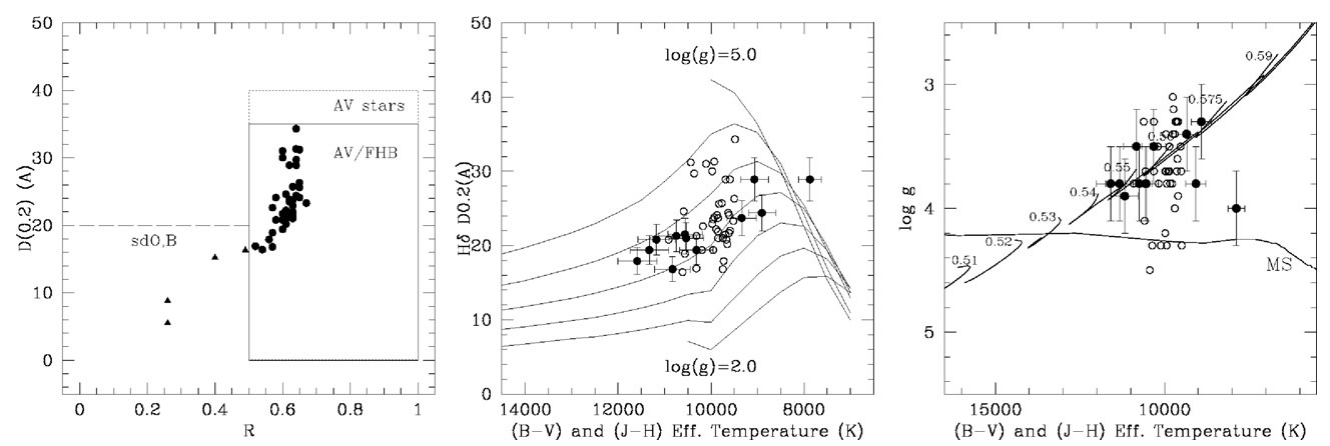

Figure 1. The various diagrams used to classify the sample. Left: $R$ versus $H \delta D_{0.2}$, which discriminates the subdwarfs, shown as filled triangles; Middle: $T_{\text {eff }}$ versus $H \delta D_{0.2}$, which separates the stars according to their $\log \mathrm{g}$; Right: $\mathrm{T}_{\text {eff }}$ versus $\log \mathrm{g}$ (HR diagram), showing the theoretical HB (for several masses, indicated) and the main sequence (MS). Filled symbols: $\mathrm{T}_{\text {eff }}$ is derived from B-V; Open symbols, $\mathrm{T}_{\text {eff }}$ estimated from J-H (Beers et al. 2007).

et al. 1992). Figure 1 (left) shows the $\mathrm{H} \delta \mathrm{D}_{0.2}$ versus $\mathrm{R}$ diagram for our sample, and the different locations occupied by the subdwarfs, FHB and Av stars. We conclude that this sample consists mainly of a mixture of FHB/Av stars with some contamination $(7-9 \%)$ of subdwarfs. However, FHB cannot be clearly separated from Av stars, because they show several similarities in their atmospheric parameters. On the other hand, it is well known that $\mathrm{FHB}$ stars with $\mathrm{T}_{\text {eff }}<10,000 \mathrm{~K}$ have lower $\log \mathrm{g}$ values when compared with main-sequence stars of the same temperature, and a distinction becomes possible if their $\log \mathrm{g}$ are derived. In this study, this is carried out comparing the position of the stars in the $\mathrm{T}_{\text {eff }}$ versus $\mathrm{H} \delta \mathrm{D}_{0.2}$ diagram with a grid of theoretical model atmospheres developed by Kurucz (Fig. 1, middle panel). The results can be best seen in the right panel, which shows the position of FHB and Av stars in a H-R diagram.

In most studies of this kind, stellar metallicities are estimated from the equivalent width of the CaII $\mathrm{K}$ line. Although this feature is moderately strong in stars with $\mathrm{T}_{\text {eff }}<9000 \mathrm{~K}$, its intensity depends strongly on the stellar temperature, which can lead to large errors if the B-V colour index is not available, for example. In this work, we verified that the MgII 4481 line can be used as an alternative to estimate $[\mathrm{Fe} / \mathrm{H}]$, because it shows little dependence with the stellar temperature, among other advantages.

\section{Conclusions}

The main results of this study can be summarized as follows: a sample containing 43 stars defined as "high-probability" FHB candidates by Beers et al. (2007) consists of $77 \%$ FHB stars, $13 \%$ main-sequence, and 10\% of subdwarfs. The MgII 4481 line consists of an alternative to the CaII $\mathrm{K}$ line for the estimation of $[\mathrm{Fe} / \mathrm{H}]$.

\section{References}

Beers, T. C., Preston, G. W., Shectman, S. A., Doinidis, S. P., \& Griffin, K. E. 1992, AJ, 103, 267

Beers, T. C., Almeida, T., Rossi, S., Wilhelm, R., \& Marsteller, B. 2007, ApJS, 168, 277

Kurucz, R. L. 1993, private communication

Ortiz, R., Malacarne, M., Wilhelm, R., Costa, R. D. D., Rossi, S., Maciel, W. J., \& Costa, A. F. M. 2007, AJ, 134, 1183

Pier, J. R. 1983, ApJS, 53, 791

Sommer-Larsen, J. \& Christensen, P. R. 1986, MNRAS, 219, 537 